\title{
Testosterona sérica e doença cardiovascular em homens
}

\author{
Serum testosterone and cardiovascular disease in men
}

Emmanuela Quental Callou de Sá', Francisco Carleial Feijó de Sá ${ }^{\text {, }}$ Alexis Dourado Guedes ${ }^{1}$, leda Therezinha do Nascimento Verreschi ${ }^{1}$

\section{RESUMO}

As doenças cardiovasculares (DCV) representam atualmente o principal grupo de causa de morte no Brasil. Os homens morrem mais de doença arterial coronariana e possuem níveis mais elevados de testosterona do que as mulheres. No entanto, estudos recentes indicam que os andrógenos podem ter efeito benéfico e/ou neutro no sistema cardiovascular masculino. Baixos níveis de testosterona endógena têm sido relacionados à presença de vários componentes da síndrome metabólica, incluindo dislipidemia, obesidade visceral, hipertensão arterial sistêmica e estados pró-trombóticos. Os dados da relação entre testosterona e reatividade vascular, aterosclerose e mortalidade cardiovascular nos homens são escassos, com os resultados de estudos disponíveis apresentando contradições. Grandes estudos randomizados e prospectivos são necessários para avaliar a função específica dos andrógenos nas DCV masculinas, para que melhores conclusões possam ser estabelecidas. Arq Bras Endocrinol Metab. 2009;53(8):915-22

\section{Descritores}

Testosterona; homens; doenças cardiovasculares; aterosclerose; doença da artéria coronariana
Disciplina de Endocrinologia e Metabologia da Escola Paulista de Medicina da Universidade Federal de São Paulo (Unifesp/ EPM), São Paulo, SP, Brasil ${ }^{1}$ Escola Paulista de Medicina da Unifesp, São Paulo, SP, Brasil 2 Instituto Dante Pazzanese de Cardiologia, São Paulo, SP, Brasil
Correspondência para:

Emmanuela Quental Callou de Sá Laboratório de Esteroides Rua Pedro de Toledo, $781,13^{\circ}$ andar 04039-032 - São Paulo, SP, Brasil eqcallou@gmail.com

Recebido em 26/Marl/2009 Aceito em 29/Abr/2009

\section{INTRODUÇÃO}

$\mathrm{A}^{\mathrm{s}}$ doenças cardiovasculares (DCV) representam, atualmente, o principal grupo de causa de morte no Brasil, sendo responsável por $31,46 \%$ do total dos óbitos; a doença cardíaca isquêmica foi responsável por $29,91 \%$ do total dessas mortes por DCV no país (Datasus, 2005).

Existe uma divergência entre as taxas de morbidade e mortalidade por DCV entre homens e mulheres que se aproximam no período pós-menopausa. Os homens morrem duas vezes mais de doença arterial coronariana (DAC), possuem maior incidência de infarto agudo do miocárdio (IAM) e níveis mais elevados de testosterona do que as mulheres. Esse dimorfismo sexual em relação às DCV pode envolver fatores genéticos, hormonais e de estilo de vida. Uma das explicações mais utilizadas para a preponderância masculina das DCV seriam os elevados níveis de testosterona sugeridos como pró-aterogênicos e/ou a ausência do efeito protetor dos estrógenos. Dessa forma, foi sugerido que os elevados níveis de 
andrógenos encontrados nos homens seriam deletérios ao sistema cardiovascular.

Excetuando-se essas evidências indiretas, foi recentemente demonstrado que a terapia de reposição com andrógenos naturais inibe a formação de ateroma em animais machos castrados (1). Além disso, estudos recentes na literatura indicam que os andrógenos endógenos podem ter um efeito benéfico e/ou neutro no sistema cardiovascular masculino.

Esta revisão sumariza evidências correntes da associação entre testosterona endógena e exógena e indicadores de doença cardiovascular nos homens. A pesquisa foi realizada na base de dados PubMed, utilizando-se os unitermos "testosterona" e "doença cardiovascular". Foram selecionados artigos em língua inglesa e portuguesa, considerando-se o delineamento experimental, a evidência clínica e a revista, de acordo com o índice de impacto.

Os níveis de evidência utilizados para graduar os estudos nessa revisão foram:

1. revisão sistemática de estudos randomizados ou estudos randomizados de grande amostra;

2. revisão sistemática de estudos não randomizados ou estudo randomizado de pequena amostra;

3. estudo de coorte não randomizado;

4. estudo retrospectivo e corte seccional;

5. opinião de especialistas, artigo de revisão não sistemática, consenso, estudos com modelos animais.

\section{MENSURAÇÃO DA TESTOSTERONA}

Mais de 95\% da testosterona circulante nos homens é secretada pelos testículos nas células de Leydig, estimuladas pela gonadotrofina luteinizante $(\mathrm{LH})$, a partir de uma série de reações enzimáticas na molécula do colesterol. No plasma, cerca de $2 \%$ da testosterona circula livre, $44 \%$ circulam ligadas à globulina ligadora de esteroides sexuais (SHBG) e $54 \%$ ligam-se à albumina e a outras proteínas. As frações livre e ligada à albumina estão prontamente disponíveis para os tecidos, sendo a junção dessas duas denominada testosterona biodisponível.

Os métodos mais largamente utilizados para a mensuração da testosterona plasmática total são o radioimunoensaio (RIA) e os ensaios por quimioluminescência, que podem ser realizados direta ou indiretamente do soro ou plasma, após extração, com ou sem cromatografia. Os métodos que incorporam extração e cromatografia são mais trabalhosos, mas apresentam várias vantagens, como separação de esteroides de reação cruzada, pro- piciando maior acurácia e sensibilidade que os ensaios diretos, no entanto, requerem validação própria (2). O padrão-ouro para a mensuração da testosterona total (T) circulante é a espectrofotometria de massa após a extração por cromatografia; o mesmo padrão-ouro para a testosterona biodisponível (TB) é a precipitação com sulfato de amônio; para a testosterona livre (TL) é a diálise de equilíbrio (2). Esses métodos, porém, são trabalhosos e caros e podem ser substituídos, na prática clínica, por técnicas indiretas de RIA para dosagem da testosterona total e fórmulas matemáticas como a de Vermeulen (3) para cálculo da testosterona biodisponível e livre:

$[\mathrm{TL}]=([\mathrm{T}]-\mathrm{N}[\mathrm{TL}]) /\left(\mathrm{K}_{\mathrm{t}}\{[\mathrm{SHBG}]-[\mathrm{T}]+\mathrm{N}[\mathrm{TL}]\}\right)$ $[\mathrm{T}]=[\mathrm{TL}]+[\mathrm{T}$ ligada à albumina $]+[\mathrm{T}$ ligada à SHBG $]$

onde:

$\mathrm{N}=\mathrm{K}_{\mathrm{a}} \mathrm{C}_{\mathrm{a}}+\mathrm{l}$;

$\mathrm{K}_{\mathrm{a}}=$ constante de associação da albumina pela $\mathrm{T}$;

$\mathrm{C}_{\mathrm{a}}=$ concentração de albumina;

$\mathrm{K}_{\mathrm{t}}=$ constante de associação da SHBG pela $\mathrm{T}$.

Apesar de episódica, a produção de testosterona apresenta um ritmo circadiano, com pico sérico pela manhã e nadir no final da tarde (4). Apresenta também sazonalidade, nos países de clima temperado com estações bem definidas, ocorrendo níveis mais elevados no final do verão e início do outono, sendo os menores no final do inverno e início da primavera (4).

Após a constatação por Vermeulen e Deslypere (5), ficou evidente que o envelhecimento nos homens é acompanhado por lento e progressivo declínio da função gonadal, manifestado pela queda da testosterona total circulante, biodisponível e livre, sendo que os níveis da testosterona biodisponível e livre caem mais acentuadamente do que a testosterona total em decorrência da elevação dos níveis de SHBG verificada com o avançar da idade.

\section{TESTOSTERONA ENDÓGENA E FATORES DE RISCO DE DOENÇAS CARDIOVASCULARES}

Baixos níveis de testosterona endógena têm sido relacionados à presença de vários componentes da síndrome metabólica, incluindo dislipidemia (Tabela 1), obesidade visceral, hipertensão arterial sistêmica (HAS) e estados pró-trombóticos (6).

Em um grande estudo prospectivo de base populacional com 1.009 homens acompanhados por 12 anos, 
a testosterona total apresentou uma correlação inversa com índice de massa corpórea (IMC), glicemia de jejum, triglicerídeos e pressão arterial sistólica e diastólica após correção para idade (7) (nível de evidência, $\mathrm{NE}=3$ ).

Em outro estudo longitudinal, com 13 anos de seguimento, níveis elevados de testosterona foram positivamente correlacionados com o HDL-colesterol (HDL-C) e associados com redução dos valores do LDL-colesterol (LDL-C) e triglicerídeos $(8)(\mathrm{NE}=3)$.

No estudo Telecom (9), que comparou homens adultos saudáveis com testosterona total plasmática normal e baixa, o grupo com níveis hormonais mais baixos apresentou maiores IMC, relação cintura-quadril, pressão arterial sistólica, glicemia e insulina de jejum e duas horas pós-sobrecarga de glicose, triglicerídeos, colesterol total e LDL-C e apolipoproteína B e menores níveis de HDL-C e apolipoproteína Al. No entanto, após correção para IMC e relação cintura-quadril, apenas a insulina de jejum, insulina pós-sobrecarga de glicose e triglicerídeos apresentaram diferença significativa entre os grupos. Nesse estudo os níveis de SHBG foram marcadamente reduzidos nos pacientes com baixos níveis de testosterona total. Os autores levantaram hipótese de causalidade para explicar a relação entre os reduzidos níveis do andrógeno e a resistência insulínica $(\mathrm{NE}=4)$.

Em uma metanálise, Ding e cols. (10) verificaram que homens com maiores níveis de testosterona tinham um risco $42 \%$ menor de diabetes melito tipo 2 . Os resultados permaneceram significativos mesmo após ajustes para raça, idade, IMC e relação cintura-quadril (NE = 2). Como mecanismo de base para essa proteção, os autores sugerem que os níveis mais elevados de testosterona aumentam a massa magra e reduzem os valores do fator de necrose tumoral- $\alpha$, melhorando, dessa maneira, a resistência insulínica.

Indivíduos morbidamente obesos e com resistência insulínica têm frequentemente baixos níveis de testosterona total, que aumentam com a perda de peso. Os mecanismos de base responsáveis pela redução dos níveis de testosterona em homens obesos não são completamente esclarecidos. A redução da testosterona livre na obesidade grave não é acompanhada por um aumento recíproco do $\mathrm{LH}$, sugerindo uma forma de hipogonadismo hipogonadotrófico. Uma hipótese postulada seria a alteração funcional no eixo hipotálamo-hipófise-testicular, caracterizada pela diminuição dos pulsos de LH (11). Um outro mecanismo sugerido seria um aumento da conversão periférica dos andrógenos em estrógenos por meio da enzima aromatase, a qual está presente em elevadas concentrações no tecido adiposo (11). Além disso, a obesidade é uma das várias condições que pode resultar em baixos níveis de SHBG, e a insulina é um importante inibidor da produção hepática dessa globulina (11). Dessa maneira, indivíduos com resistência insulínica tendem a apresentar valores mais baixos de testosterona total.

Os adipócitos expressam receptores androgêni$\cos$ (RA) (12). Os andrógenos ativam os receptores $\beta$-adrenérgicos e a lipase hormônio sensível, além de inibirem a atividade da lipase lipoproteica, a principal enzima reguladora da captação de triglicerídeos no tecido adiposo (12). Como resultado, a testosterona estimula a lipólise e reduz o estoque de gordura no tecido adiposo, sendo o efeito inverso, aumento da massa gorda corporal, observado em pacientes hipogonádicos.

\section{TESTOSTERONA EXÓGENA E FATORES DE RISCO DAS DOENÇAS CARDIOVASCULARES}

As formulações de testosterona disponíveis para uso clínico são intramuscular (IM), transdérmica, bucal, oral e para implante subcutâneo, sendo a primeira a mais amplamente utilizada.

A testosterona livre injetável não esterificada tem uma meia-vida do hormônio livre de somente dez minutos; já os ésteres de testosterona apresentam meia-vida mais prolongada (13). As desvantagens dos ésteres é que eles inicialmente fornecem níveis suprafisiológicos de testosterona, os quais declinam lentamente e, antes da próxima aplicação, níveis reduzidos já podem ser encontrados (efeito montanha-russa) (13).

Os esteroides são facilmente absorvidos através da pele. As preparações transdérmicas estão disponíveis nas formas de adesivos escrotais e não escrotais e, mais recentemente, como gel (14). As modalidades transdérmicas, em comparação com todas as outras preparações disponíveis, fornecem níveis séricos de testosterona mais fisiológicos (13).

Quando administrada oralmente na forma livre, a testosterona é bem absorvida pelo intestino, porém, é metabolizada e inativada pelo fígado antes de atingir os órgãos alvos (efeito de primeira passagem) (13). Somente com doses elevadas, a capacidade de metabolização hepática é ultrapassada (13). Várias tentativas foram realizadas para modificar a molécula de testosterona e torná-la mais efetiva oralmente. Os derivados 17- $\alpha$-alquilados, apesar de efetivos, apresentam efeitos 
colaterais, tais como elevação das enzimas séricas do fígado, peliosis, colestase e tumores hepáticos (13). O undecanoato de testosterona é absorvido pelos linfáticos intestinais e alcança a circulação via ducto torácico, escapando do efeito de primeira passagem hepática (13). No entanto, os níveis séricos da testosterona variam largamente com essa terapia, limitando seu uso (13).

Um modelo de mucoadesivo bucal de testosterona tem sido desenvolvido para o tratamento do hipogonadismo masculino, comercializado pelo nome de Striant nos Estados Unidos (15). Parece ser uma forma de apresentação bem tolerada, com pequena incidência de efeitos colaterais (15).

Os implantes de testosterona subcutânea fornecem aos pacientes níveis plasmáticos normais do hormônio por três a seis meses (16). No entanto, seu uso não se encontra amplamente difundido na prática clínica.

Os efeitos da testosterona exógena nos fatores de risco das DCV podem depender da via de administração, duração do tratamento e idade. Os resultados dos estudos não são uniformes, sendo verificada a discordância entre eles, principalmente em relação à influência da reposição da testosterona sobre os níveis séricos do HDL-C.

Em uma metanálise publicada por Whitsel e cols. (17), a administração de testosterona IM esteve relacionada à redução dos valores do HDL-C. Nos pacientes mais idosos e com período mais longo de tratamento, esse efeito na diminuição do HDL-C foi menos proeminente $(\mathrm{NE}=2)$. Em estudos conduzidos por Snyder e cols. (18) e Howell e cols. (19), não foram observadas diferenças no perfil lipídico em pacientes hipogonádicos tratados com testosterona transdérmica $(\mathrm{NE}=3 \mathrm{e}$ 2 , respectivamente).

Dobs e cols. (20) verificaram que, em homens hipogonádicos, a reposição de testosterona, tanto transdérmica quanto IM, provocou redução do HDL-C e aumento da relação colesterol total/HDL-C. A testosterona IM, mas não a transdérmica, diminuiu os valores do colesterol total quando comparados ao estado hipogonádico. Não houve diferença no perfil lipídico entre as duas apresentações $(\mathrm{NE}=3)$.

Os estudos já citados no presente trabalho sobre os efeitos da reposição da testosterona nos fatores de risco das DCV apresentam características que merecem considerações. Os estudos conduzidos por Snyder e cols. (18) e por Dobs e cols. (20) são cortes prospectivos com pequeno número de participantes, já o conduzido por Howell e cols. (19), apesar de randomizado, apresenta igualmente reduzido número de sujeitos, além de ser cego apenas para os pacientes. Por fim, a metanálise publicada por Whitsel e cols. (17) apresenta 272 participantes, provenientes de 19 estudos, sendo alguns deles observacionais.

Diferentemente dos estudos citados e em colaboração com membros da The Endocrine Society para Força-Tarefa na Testosterona em Homens com Deficiência Androgênica, Haddad e cols. (21), em metanálise de 30 estudos randomizados, placebos-controlados, que incluiu 1.642 homens, 808 dos quais tratados com testosterona nas formulações disponíveis comercialmente (IM, transdérmica, oral e bucal), evidenciaram efeitos negligenciáveis da utilização da testosterona nas frações lipídicas, pressão arterial e controle glicêmico $(\mathrm{NE}=1)$.

Dada a escassez de estudos com a qualidade metodológica de Haddad e cols. (21), faz-se ainda necessário que pesquisadores avaliem a relação entre testosterona exógena e fatores de risco das DCV.

Verificada a importância da formação de trombos na patogênese de eventos coronarianos agudos, têm sido identificados vários fatores hemostáticos como sendo de risco de DCV. O risco de DAC é influenciado positivamente pela agregabilidade plaquetária e níveis de fatores trombogênicos, como PAI-1, fator VII e fi-

\begin{tabular}{|c|c|c|c|c|c|}
\hline Estudo & Desenho & $\mathbf{n}$ & Via de administração da T & Efeitos no perfil lipídico & NE \\
\hline Whitsel e cols. (17) & Metanálise & 272 & IM & $\downarrow$ HDL, restante nulo & 2 \\
\hline Snyder e cols. (18) & Coorte prospectivo & 16 & TTD & nulo & 3 \\
\hline Howell e cols. (19) & Randomizado, placebo-controlado & 33 & TTD & $\downarrow$ LDL, restante nulo & 2 \\
\hline Dobs e cols. (20) & Coorte prospectivo & 29 & IM/TTD & $\begin{array}{l}\text { IM - } \downarrow \mathrm{CT}, \downarrow \mathrm{HDL}, \uparrow \mathrm{CT} / \mathrm{HDL} \text {, restante nulo } \\
\text { TTD - } \downarrow \text { HDL, } \uparrow \mathrm{TG}, \uparrow \mathrm{CT} / \mathrm{HDL} \text {, restante nulo }\end{array}$ & 3 \\
\hline Haddad e cols. (21) & Metanálise & 1.642 & IM/TTD/oral/bucal & $\begin{array}{l}\text { T normal - } \downarrow \text { CT, restante nulo } \\
\text { Hipogonádicos - nulo }\end{array}$ & 1 \\
\hline
\end{tabular}

N: número de sujeitos; T: testosterona; NE: nível de evidência; IM : intramuscular; TTD: testosterona transdérmica; CT: colesterol total; TG: triglicerídeos. 
brinogênio. Em estudo conduzido por Philips e cols. (22), os valores de testosterona circulante correlacionaram-se negativamente com fibrinogênio e fator VII em pacientes com $\mathrm{DAC}(\mathrm{NE}=4)$. A administração de testosterona a 32 homens saudáveis, participantes de um estudo de contracepção masculina, provocou uma diminuição sustentada do fibrinogênio em $15 \%$ a $20 \%$ (23) $(\mathrm{NE}=3)$. Por outro lado, a reposição de testosterona exógena tem demonstrado aumentar a agregabilidade plaquetária $(24)(\mathrm{NE}=2)$. Dessa forma, a reposição de testosterona mostra resultados tanto pró-fibrinolíticos quanto pró-agregatórios, sendo sua influência na formação de trombos o resultado do balanço desses dois efeitos.

\section{TESTOSTERONA E REATIVIDADE VASCULAR}

Os RA distribuem-se amplamente pelo sistema cardiovascular como a aorta, a vasculatura periférica, as células atriais e ventriculares, as células endoteliais, as plaquetas e os megacariócitos (25).

A testosterona pode exercer seus efeitos cardiovasculares direta e indiretamente, após a conversão em estradiol $\left(\mathrm{E}_{2}\right)$. As células endoteliais, musculares lisas, macrófagos e plaquetas expressam aromatase e 17- $\beta$-hidroxiesteroide desidrogenase, permitindo que $\mathrm{O}_{2}$ seja produzido localmente, a partir de precursores circulantes como testosterona e DHEA(26).

A testosterona pode ainda exercer efeitos no sistema cardiovascular por modelos genômicos, por meio dos RA e não genômicos, pela sua ligação a receptores de esteroides na membrana plasmática - o que pode acarretar bloqueio dos canais $\mathrm{L}$ de cálcio e abertura dos canais de potássio ativados por cálcio (27).

A variação do número de repetições CAG no éxon 1 do gene do receptor androgênico é inversamente relacionada à resposta transcricional à testosterona $(28)$. Em um estudo com 110 homens saudáveis, foi observada uma associação positiva entre o número dessas repetições e a vasodilatação por mecanismos dependentes e independentes do endotélio (29) $(\mathrm{NE}=4)$. Dessa forma, uma maior sensibilidade à testosterona, mediada por modelo de ação genômico, prejudicaria a vasodilatação arterial.

Acredita-se que, por mecanismos não genômicos, a testosterona possa atuar como vasodilatador coronariano. Em um estudo conduzido por Rosano e cols. (30), a administração de $2,5 \mathrm{mg}$ de testosterona $\mathrm{EV}$ em homens com DAC prolongou o tempo para início da depressão de $1 \mathrm{~mm}$ do segmento ST no teste de esforço e o tempo total do exercício, quando comparado ao placebo $(\mathrm{NE}=2)$. Em um outro estudo randomizado, duplo-cego, placebo-controlado, com 46 homens portadores de angina crônica estável, a suplementação com $5 \mathrm{mg} /$ dia de testosterona transdérmica, por um período de 12 semanas, melhorou o limiar de isquemia, quando comparado ao placebo $(31)(\mathrm{NE}=2)$.

Contrariamente ao exposto anteriormente sobre a melhora na DAC, a administração de $200 \mathrm{mg}$ de cipionato de testosterona IM a homens saudáveis em um estudo randomizado, duplo-cego e placebo-controlado aumentou a agregação plaquetária por modular a expressão dos receptores de tromboxano $(24)(\mathrm{NE}=2)$. Em outro estudo, a testosterona foi implicada em prejudicar a vasodilatação coronariana em resposta à adenosina em modelos isolados de coração de rato (32) $(\mathrm{NE}=5)$. Tal efeito foi mediado agudamente e atribuído à liberação de tromboxano, o qual é um agente vasoconstritor e agregante plaquetário, que tem sido implicado na patogênese da DCV.

Em resumo, os efeitos vasculares da testosterona, dessa forma, seriam provavelmente decorrentes do balanço entre vasodilatação e vasoconstrição.

\section{TESTOSTERONA E ATEROSCLEROSE}

Aterosclerose é uma palavra de origem grega, que se refere ao espessamento da camada íntima da artéria (sclerosis, "endurecimento") e ao acúmulo de lipídeos nesta (athere, "papa").

Os dados da relação entre testosterona e aterosclerose são escassos, com resultados contraditórios dos estudos disponíveis. Além disso, em alguns destes não foi referido o horário da coleta hormonal ou este foi bastante amplo. Tendo em vista que a testosterona é um hormônio com reconhecido ritmo circadiano, os resultados de alguns estudos devem ser interpretados com cautela diante dessa limitação (Tabela 2).

Hak e cols. (33), em um estudo de base populacional, encontraram uma relação inversa e independente entre os níveis de testosterona total e biodisponível, com aterosclerose aórtica grave e progressão desta, verificadas por radiografia de coluna lombar $(\mathrm{NE}=3)$.

Tivesten e cols. (34) não encontraram correlação estatística entre testosterona total e livre e progressão da aterosclerose de carótidas em 313 homens saudáveis com 58 anos de idade, habitantes de Gothenburg, Suécia $(\mathrm{NE}=3)$. 


\begin{tabular}{|c|c|c|c|c|c|c|c|}
\hline Estudo & Desenho & $\mathbf{n}$ & $\mathbf{T}$ & A & Efeito na $A$ & $\begin{array}{c}\text { Horário de coleta } \\
\text { da T }\end{array}$ & NE \\
\hline Hak e cols. (33) & $\begin{array}{c}\text { Coorte prospectivo de base } \\
\text { populacional }\end{array}$ & 504 & $\mathrm{TT} / \mathrm{TB}$ & Aórtica & $\downarrow / \downarrow$ & 08:30-16:00 & 3 \\
\hline Tivesten e cols. (34) & Coorte prospectivo & 313 & $\mathrm{TT} / \mathrm{TL}$ & Carótida & Nulo/nulo & Período da manhã & 3 \\
\hline Tivesten e cols. (35) & Corte seccional de base populacional & 2.784 & $\mathrm{TT} / \mathrm{TL}$ & MMII & $\downarrow / \downarrow$ & Não referido & 4 \\
\hline Philips e cols. (22) & Corte seccional & 55 & $\mathrm{TT} / \mathrm{TL}$ & Coronária & $\downarrow / \downarrow$ & Não referido & 4 \\
\hline English e cols. (36) & Corte seccional & 90 & $\mathrm{TT} / \mathrm{TB} / \mathrm{TL} /$ & Coronária & Nulo/ $/ /$ nulo & 08:30-09:30 & 4 \\
\hline Harman e cols. (37) & Coorte prospectivo & 890 & $\mathrm{TT} / \mathrm{TL}$ & Coronária & Nulo/nulo & 07:00-09:30 & 3 \\
\hline
\end{tabular}

N: número de sujeitos; T: testosterona; A: aterosclerose; NE: nível de evidência; TT: testosterona total; TB: testosterona biodisponível; TL: testosterona livre.

Posteriormente, em outro estudo conduzido por Tivesten e cols. (35), a relação entre testosterona total e livre e doença arterial periférica de membros inferiores foi avaliada em 2.784 homens idosos. Os autores verificaram que testosterona total e livre correlacionaram-se positivamente com o índice tornozelo-braço, indicando uma associação negativa entre esse andrógeno e a doença aterosclerótica das extremidades inferiores $(\mathrm{NE}=4)$. Esse índice constitui-se em uma razão da pressão arterial do tornozelo e do braço, com o paciente em posição supina; um valor baixo indica a presença de doença arterial das extremidades inferiores.

A relação entre testosterona e aterosclerose coronariana também foi estudada por vários autores. Philips e cols. (22) descreveram correlação negativa entre testosterona total e livre e DAC em 55 homens sem história prévia de IAM $(\mathrm{NE}=4)$. English e cols. $(36)$, em um estudo caso-controle (60 casos e 30 controles), encontraram uma associação negativa entre testosterona biodisponível, livre e índice de andrógenos livres (IAL) com DAC. Após a correção para idade e IMC, persistiu a correlação negativa com testosterona livre e IAL (NE =4). Contrariamente aos resultados já citados, Harman e cols. (37), em estudo prospectivo longitudinal com 890 homens, com média de idade 53,8 \pm 16 anos e seguidos por um período superior a 31 anos, não verificaram correlação entre os níveis basais de testosterona total e livre e o subsequente desenvolvimento de DAC $(\mathrm{NE}=3)$.

\section{TESTOSTERONA E MORTALIDADE CARDIOVASCULAR}

A associação entre testosterona e mortalidade cardiovascular também tem despertado o interesse de vários pesquisadores, e numerosos estudos vêm sendo publi- cados sobre o tema. Da mesma forma que se observa a partir dos assuntos abordados anteriormente, controvérsias existem na literatura, e estudos adicionais são necessários para avaliação dessa associação (Tabela 3).

Em um estudo prospectivo com 12 anos de acompanhamento, com 1.009 homens com idade entre 40 e 79 anos, residentes da comunidade de Rancho Bernardo, na Califórnia, não foi encontrada correlação estatística entre níveis basais de testosterona total e subsequente desenvolvimento de morbidade e/ou mortalidade por DCV $(7)(\mathrm{NE}=3)$. Posteriormente, nessa mesma comunidade, num período médio de acompanhamento de 11,8 anos, foi verificado em 794 homens idosos que aqueles com menores níveis de testosterona total e biodisponível apresentavam maior mortalidade por $\operatorname{DCV}(38)(\mathrm{NE}=3)$. Neste último estudo, a média de idade no início do seguimento foi de 71,2 anos e taxa de mortalidade, $68 \%$, o que poderia justificar, em parte, a diferença entre os resultados.

Em um estudo prospectivo de caso-controle, de pacientes saudáveis entre 40 e 79 anos de idade e período médio de acompanhamento de sete anos, concentrações mais elevadas de testosterona endógena estiveram inversamente relacionadas à mortalidade geral e por causas cardiovasculares (39) $(\mathrm{NE}=3)$.

Araujo e cols. (40), em estudo de corte prospectivo de base populacional, com 1.686 homens entre $40 \mathrm{e}$ 70 anos de idade e seguidos por um período médio de 15,3 anos, não encontraram relação estatística entre valores de testosterona total e mortalidade por DAC. No entanto, foi verificada uma associação positiva e outra negativa entre testosterona livre $(\mathrm{p}=0,02)$ e diidrotestosterona $(\mathrm{p}=0,04)$ e mortalidade por DAC, respectivamente. Duas amostras séricas foram coletadas de cada indivíduo participante, com intervalo de 30 minutos, quatro horas após terem acordado $(\mathrm{NE}=3)$. Em um 
Tabela 3. Associação entre testosterona e mortalidade cardiovascular

\begin{tabular}{|c|c|c|c|c|c|c|}
\hline Estudo & Desenho & $\mathbf{n}$ & $\begin{array}{l}\text { Seguimento } \\
\text { (anos) }\end{array}$ & $\mathbf{T}$ & $\begin{array}{c}\text { Associação com } \\
\text { mortalidade por DCV }\end{array}$ & NE \\
\hline Barret-Connor e Khaw (7) & Prospectivo de base populacional & 1.009 & 12 & TT & Nulo & 3 \\
\hline Laughlin e cols. (38) & Prospectivo de base populacional & 794 & 11,8 & $\mathrm{TT} / \mathrm{TB}$ & $\downarrow / \downarrow$ & 3 \\
\hline Khaw e cols. (39) & Prospectivo caso-controle & 2.314 & 7 & $\mathrm{TT}$ & $\downarrow$ & 3 \\
\hline Araujo e cols. (40) & Prospectivo de base populacional & 1.686 & 15,3 & TT & Nulo & 3 \\
\hline Smith e cols. (41) & Prospectivo de base populacional & 2.512 & 16,5 & $\mathrm{TT} / \mathrm{TL} / \mathrm{DHT}$ & Nulo/ $/ / \downarrow$ & 3 \\
\hline
\end{tabular}

N: número de sujeitos; T: testosterona; DCV: doença cardiovascular; NE: nível de evidência; TT: testosterona total; TB: testosterona biodisponível; TL: testosterona livre; DHT: diidrotestosterona.

outro estudo prospectivo, de base populacional, conduzido por Smith e cols. (41), com 2.512 homens entre 45 e 59 anos de idade e seguidos por um período médio de 16,5 anos, não foi encontrada correlação estatística entre testosterona total e mortalidade por todas as causas e por $\mathrm{DAC}(\mathrm{NE}=3)$.

\section{CONCLUSÕES}

Com o envelhecimento da população, tem-se observado aumento na prevalência das DCV, e a necessidade da avaliação de novos fatores de risco ditos não clássicos tem emergido. Nesse ínterim, o interesse do papel da testosterona na patologia cardiovascular vem crescendo de forma acentuada. À luz dos conhecimentos atuais, a testosterona endógena exerce um efeito neutro e/ou benéfico no sistema cardiovascular ( $\mathrm{SCV}$ ) masculino. No que se refere à testosterona exógena, as incertezas aumentam, e os resultados dos estudos são bastante variáveis na dependência dos valores basais desse hormônio no momento da reposição, da dose administrada e da idade do paciente.

No entanto, os desfechos dos estudos não são homogêneos. Em vários deles podem ser verificadas falhas metodológicas graves. Inúmeros apresentam um pequeno número de participantes, e a grande maioria utiliza populações selecionadas. Faz-se necessário que novos estudos avaliem o papel da testosterona na DCV nos homens, especialmente os prospectivos, randomizados e multicêntricos, para que conclusões mais precisas sobre o papel desse andrógeno no SCV masculino possam ser retiradas.

Agradecimentos: os autores agradecem o incentivo da disciplina de Endocrinologia e Metabologia da Escola Paulista de Medicina da Universidade Federal de São Paulo (Unifesp/EPM) para o desenvolvimento desta revisão, especialmente a Doutora Marise Lazaretti Castro.

Declaração: os autores declaram não haver conflitos de interesse científico neste estudo.

\section{REFERÊNCIAS}

1. Alexandersen P, Haarbo J, Byrjalsen I, Lawaetz H, Christiansen C. Natural androgens inhibit male atherosclerosis: A study in castrated, cholesterol fed rabbits. Circ Res. 1999;84(7):813-9.

2. Rosner W, Auchus RJ, Azziz R, Sluss PM, Raff H. Position statement: Utility, limitations, and pitfalls in measuring testosterone: an Endocrine Society position statement. J Clin Endocrinol Metab. 2007;92(2):405-13.

3. Vermeulen A, Verdonck L, Kaufman JM. A critical evaluation of simple methods for the estimation of free testosterone in serum. J Clin Endocrinol Metab. 1999;84(10):3666-72.

4. Kapoor D, Malkin CJ, Channert KS, Jones TH. Androgens, insulin resistance and vascular disease in men. Clin Endocrinol (Oxf). 2005;63(3):239-50.

5. Vermeulen A, Deslypere JP. Testicular endocrine function in the ageing male. Maturitas. 1985;7(3):273-9.

6. Malkin CJ, Pugh PJ, Jones TH, Channer KS. Testosterone for secondary prevention in men with ischaemic heart disease? OJM. 2003;96(7):521-9.

7. Barret-Connor E, Khaw KT. Endogenous sex hormones and cardiovascular disease in men. A prospective population-based study. Circulation. 1988;78(3):539-45.

8. Zmuda JM, Cauley JA, Kriska A, Glynn NW, Gutai JP, Kuller LH. Longitudinal relation between endogenous testosterone and cardiovascular disease risk factors in middle-aged men. A 13 year follow-up of former multiple risk factors intervention trial participants. Am J Epidemiol. 1997;146(8):609-17.

9. Simon D, Charles MA, Nahoul K, Orssaud G, Kremski J, Hully V, et al. Association between plasma total testosterone and cardiovascular risk factors in healthy adult men:The Telecom Study. J Clin Endocrinol Metab. 1997;82(2):682-5.

10. Ding EL, Song Y, Malik VS, Liu S. Sex differences of endogenous sex hormones and risk of type diabetes: a systematic review and meta-analysis. JAMA. 2006;295(11):1288-99.

11. Vermeulen A. Decreased androgen levels and obesity. Ann Med. 1996;28(1):13-5.

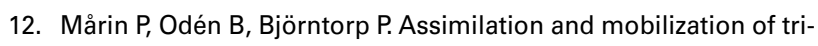
glycerides in subcutaneous abdominal and femoral adipose tissue in vivo in men: effects of androgens. J Clin Endocrinol Metab. 1995;80(1):239-43.

13. Nieschlag E, Behre HM. Pharmacology and clinical uses of testosterone. In: Nieschlag E, Behre HM, editors. Testosterone: action, deficiency, substitution. 2. ed. Berlin Heidelberg: Springer-Verlag; 1998. p. 293-328.

14. Rhoden EL, Morgentaler A. Risks of testosterone-replacement therapy and recommendations for monitoring. $\mathrm{N}$ Engl $\mathrm{J}$ Med. 2004;350(5):482-92.

15. Korbonits M, Kipnes M, Grossman AB. Striant SR: a novel, effective and convenient testosterone therapy for male hypogonadism. Int J Clin Pract. 2004;58(11):1073-80. 
16. Gooren LJ, Bunck MC. Androgen replacement therapy: present and future. Drugs. 2004;64(17):1861-91.

17. Whitsel EA, Boyko EJ, Matsumoto AM, Anawalt BD, Siscovick DS. Intramuscular testosterone esters and plasma lipids in hypogonadal men: a meta-analysis. Am J Med. 2001;111(4):261-9.

18. Snyder PJ, Peachey H, Berlin JA, Hannoush P, Haddad G, Dlewati $A$, et al. Effects of testosterone replacement in hypogonadal men. J Clin Endocrinol Metab. 2000;85(8):2670-7.

19. Howell SJ, Radford JA, Admas JE, Smets EMA, Warburton R, Shalet SM. Randomized placebo-controlled trial of testosterone replacement in men with mild Leydig cell insufficiency following cytotoxic chemotherapy. Clin Endocrinol (Oxf). 2001;86:4078-88.

20. Dobs AS, Bachorik PS, Arver S, Meikle AW, Sanders SW, Caramelli KE, et al. Interrelationships among lipoprotein levels, sex hormones, anthropometric parameters, and age in hypogonadal men treated for 1 year with a permeation-enhanced testosterone transdermal system. J Clin Endocrinol Metab. 2001;86(3):1026-33.

21. Haddad RM, Kennedy CC, Caples SM, Tracz ML, Boloña ER, Siders $\mathrm{K}$, et al. Testosterone and cardiovascular risk in men: a systematic review and meta-analysis of randomized placebo-controlled trials. Mayo Clin Proc. 2007;82(1):29-39.

22. Philips GB, Pinkernell BH, Jing TY. The association of hypotestosteronemia with coronary artery disease in men. Arterioscler Thromb Vasc Biol. 1994;14(5):701-6.

23. Anderson RA, Ludlam CA, Wu FCW. Haemostatic effects of supraphysiological levels of testosterone in normal men. Thromb Haemost. 1995;74(2):693-7.

24. Ajayi AA, Marthur R, Haluska PV. Testosterone increase human platelet tromboxane $A_{2}$ receptor density and aggregation responses. Circulation. 1995;91(11):2742-7.

25. Ruizeveld de Winter JA, Trapman J, Vermey M, Mulder E, Zegers ND, van der Kwast TH. Androgen receptor expression in human tissues: an immunohistochemical study. J Histochem Cytochem. 1991;39(7):927-36

26. Harada N, Sasano H, Murakami H, Ohkuma T, Nagura H, Takagi Y. Localized expression of aromatase in human vascular tissue. Cir Res. 1999;84(11):1285-91.

27. Webb CM, McNeill JG, Hayward CS, de Zeigler D, Collins P. Effects of testosterone on coronary vasomotor regulation in men with coronary heart disease. Circulation. 1999;100(16):1690-6.

28. Beilin J, Ball EM, Favaloro JM, Zajac JD. Effect of the androgen receptor CAG repeat polymorphism on transcriptional activity: specificity in prostate and non-prostate cell lines. J Mol Endocrinol. 2000;25(1):2585-96.

29. Zitzmann M, Brune M, Kornmann B, Gromoll J, von Eckardstein $S$, von Eckardstein $A$, et al. The CAG repeat polymorphism in the
AR gene affects high density lipoprotein cholesterol and arterial vasoreactivity. J Clin Endocrinol Metab. 2001;86(10):4867-73.

30. Rosano GMC, Leonardo F, Pagnotta P, Pelliccia F, Panina G, Cerquetani $E$, et al. Acute anti-ischemic effect of testosterone in men with coronary artery disease. Circulation. 1999;99(13):1666-70.

31. English KM, Steeds RP, JonesTH, Diver MJ, Channer KS. Low-dose transdermal testosterone therapy improves angina threshold in men with chronic stable angina. Circulation. 2000;102(16):1906-11.

32. Ceballos G, Figueroa L, Rubio I, Gallo G, Garcia A, Martinez A, et al. Acute and nongenomic effects of testosterone on isolated and perfused rat heart. J Cardiovasc Phamarcol. 1999;33(5):691-7.

33. Hak AE, Witteman JCM, Jong FH, Geerlings MI, Hofman A, Pols HAP. Low levels of endogenous androgens increase the risk of atherosclerosis in elderly men: The Rotterdam Study. J Clin Endocrinol Metab. 2002;87(8):3632-9.

34. Tivesten A, Huthle J, Wallenfeldt K, Wikstrand J, Ohlsson C, Fagerberg $B$. Circulation estradiol is an independent predictor of progression of carotid artery intima-media thickness in middleaged men. J Clin Endocrinol Metab. 2006;91(11):4433-7.

35. Tivesten A, Mellström D, Jutberger $H$, Fagerberg B, Lernfelt $B$, Orwoll $E$, et al. Low serum testosterone and high serum estradiol associate with lower extremity peripheral arterial disease in elderly men:The MrOS Study in Sweden. J Am Coll Cardiol. 2007;50(11):1077-9.

36. English KM, Mandour O, Steeds RP, Diver MJ, JonesTH, Channer KS. Men with coronary artery disease have lower level of androgens than men with normal coronary angiograms. Eur Heart J. 2000;21(11):890-4

37. Harman SM, Metter EJ, Tobin JD, Pearson J, Blackman MR; Baltimore Longitudinal Study of Aging. Longitudinal effects of aging on serum total and free testosterone levels in healthy men. J Clin Endocrinol Metab. 2001;86(2):724-31.

38. Laughlin GA, Barret-Connor E, Bergstrom J. Low serum testosterone and mortality in older men. J Clin Endocrinol Metab. 2008;93(1):68-75.

39. Khaw KT, Dowsett M, Folkerd E, Bingham S, Wareham N, Luben R, et al. Endogenous testosterone and mortality due to all causes, cardiovascular disease, and cancer in men: European prospective investigation into cancer in Norfolk (EPIC-Norfolk) Prospective Population Study. Circulation. 2007;116(23):2694-701.

40. Araujo AB, Kupelian V, Page ST, Handelsman DJ, Bremner WJ, Mckinlay JB. Sex steroids an all-cause and cause-specific mortality in men. Arch Intern Med. 2007;167(12):1252-60.

41. Smith GD, Ben-Shlomo Y, Beswick A, Yarnell J, Lightman S, Elwood P. Cortisol, testosterone, and coronary heart disease: prospective evidence from the Caerphilly study. Circulation. 2005;112(3):332-40. 